\title{
Postoperative conjunctival chemosis in cataract surgery caused by subconjunctival gentamicin injection
}

\author{
Milind Pande
}

\begin{abstract}
A prospective study of 75 patients undergoing cataract surgery was conducted to identify the cause of postoperative conjunctival chemosis seen in patients with cataracts in this department. The patients were split in three groups of 25 each. Group G patients were given a subconjunctival injection of gentamicin at the end of the cataract procedure; group $W$ patients did not receive subconjunctival gentamicin, but a cellulose wick drain was used during the procedure; and Group $\mathbf{G W}$ patients received a subconjunctival injection of gentamicin and a wick drain was also used. The incidence, severity, and the duration of chemosis were observed in the three groups. Chemosis was seen only in groups $G$ and $G W$, and the difference between the number of patients who received gentamicin and those who did not receive gentamicin was significant $(\mathbf{p}<0.001)$.
\end{abstract}

Postoperative conjunctival chemosis was observed in a large proportion of our patients undergoing extracapsular cataract extraction and posterior chamber implantation procedures in the early part of 1989 . This chemosis was always inclusive and invariably restricted to the lower fornicial and bulbar conjunctiva. The severity varied from a mild involvement of the lower fornicial conjunctiva to a gross chemosis extending into the upper bulbar conjunctiva with loose chemosed conjunctiva in the palpebral fissure. There was capillary closure in the conjunctival vasculature overlying the chemosed area (Fig 1).

The fact that the lower half of the conjunctiva

Department of Ophthalmology, Hull Royal Infirmary, Hull HU3 2JZ

$M$ Pande

Correspondence to:

Mr M Pande, FRCS.

Accepted for publication 4 April 1991
Figure 1 Slit-lamp photograph showing capillary cl the overlying conjunctival vasculature in chemosed lor fornicial and bulbar conjunctiva. was always involved pointed to two surgical events occurring exclusively in this area. One was the insertion of a cellulose wick to drain fluid away from the operative field during the operation, and the other was the subconjuctival injection of gentamicin at the end of the operation. This prospective study was set up to investigate whether one or both of these surgical events were giving rise to the chemosis.

\section{Patients and methods}

Patients undergoing extracapsular cataract extractions between 21 September 1989 and 19 January 1990 at the Hull Royal Infirmary were included. Their ages ranged from 44 to 88 years (mean 74.67 years). A total of 75 eyes of 75 patients were enrolled into this study.

Patients who had a past history of conjunctival chemosis, chemical burn, conjunctival trauma, keratoconjunctivitis sicca, and conjunctival lesions in the form of scarring and symblepharon were excluded from this study.

The patients were all waiting list admissions and were sequentially allocated on admission into three groups as follows. Each group consisted of 25 patients. Group $G$. Patients given a subconjunctival injection of $20 \mathrm{mg}$ of gentamicin (Cidomycin) in the lower fornix at the end of surgery. Wick was not used during surgery. Group $W$. A wick was inserted into the lower fornix during surgery. Patients did not get a conjunctival injection at the end of the operation. Group $G W$. A wick and a subconjunctival injection were used as described above.

All patients underwent endocapsular or extracapsular cataract surgery under general anaesthesia by three surgeons using a manual aspiration technique. The duration of wick contact was recorded in patients of groups W and $\mathrm{GW}$, and a $20 \mathrm{mg}$ subconjunctival injection of gentamicin was given to patients in groups $\mathrm{G}$ and GW.

The conjunctival sacs of patients were examined and the presence or absence of chemosis was noted. If present the severity was noted in accordance with the following criteria: grade 1 - involvement limited to $10 \%$ to $30 \%$ of the conjunctiva; grade 2 - involvement limited to $30 \%$ to $70 \%$ to the conjunctiva; grade 3 involvement limited to $70 \%$ to $100 \%$ of the conjunctiva.

The patients were examined daily while on the ward and the trend of chemosis noted. They were again examined for chemosis on their first postoperative clinic visit. During his examination of them the observer did not know to which groups the patients had been allocated. 
Table 1 Incidence of chemosis in the study groups

\begin{tabular}{llcl}
\hline & \multicolumn{4}{l}{ Number of patients $(\%)$} \\
\cline { 2 - 4 } Group & Chemosis & No chemosis & Total \\
\hline $\mathrm{G}$ & $23(92)$ & $2(8)$ & 25 \\
$\mathrm{~W}$ & $0(0)$ & $25(100)$ & 25 \\
$\mathrm{GW}$ & $22(88)$ & $3(12)$ & 25 \\
\hline
\end{tabular}

Table 2 Severity of chemosis in the study groups

\begin{tabular}{llllll}
\hline \multirow{5}{*}{ Number of patients $(\%)$} \\
\cline { 2 - 6 } & Grade 1 & Grade 2 & Grade 3 & No chemosis & Total \\
\hline G & $7(28)$ & $13(52)$ & $3(12)$ & $2(8)$ & 25 \\
W & $0(0)$ & $0(0)$ & $0(0)$ & $25(100)$ & 25 \\
GW & $6(24)$ & $13(52)$ & $3(12)$ & $3(12)$ & 25 \\
\hline
\end{tabular}

\section{Results}

Table 1 shows the results of the incidence of chemosis in the three groups. Twenty three $(92 \%)$ of the 25 cases in group G, none of the 25 cases in group $\mathrm{W}$, and $22(88 \%)$ of the 25 cases in group GW had chemosis. The incidence of chemosis in group $\mathrm{G}$ and group W clearly suggests subconjunctival gentamicin as the cause of the chemosis. The difference in the incidence of chemosis in the groups in which subconjunctival gentamicin was used (groups G and GW) and the group in which no subconjunctival gentamicin was used is significant at the 0.001 level by the $\chi^{2}$ test.

Table 2 shows the severity of chemosis in the three groups. In group $\mathrm{G}$ of the 25 cases 7 (28\%) had grade 1 chemosis, $13(52 \%)$ had grade 2 chemosis, $3(12 \%)$ had grade 3 chemosis, and 2 $(8 \%)$ had no chemosis. In group GW of the 25 cases $6(24 \%)$ had grade 1 chemosis, $13(52 \%)$ had grade 2 chemosis, $3(12 \%)$ had grade 3 chemosis, and $3(12 \%)$ had no chemosis. In group W none of the 25 cases had chemosis. There is no statistically significant difference in the incidence of the three grades of chemosis between groups $\mathrm{G}$ and $\mathrm{GW}$, indicating that the use of the wick did not influence the severity of chemosis in group GW. The conclusion is strongly supported by the fact that no chemosis was seen in any of the cases in group W, where only the wick was used. Table 3 shows the duration of chemosis seen in the 45 patients in groups $G$ and $G W$ who had chemosis. The duration of chemosis in these 45 patients ranged from three to seven days, average 4.64 days.

\section{Discussion}

The cause of postoperative conjunctival chemosis in this study was the use of subconjunctival gentamicin injection at the end of the cataract operation. The cellulose wick drain did not influence the chemosis in any way.

The prophylactic use of subconjunctival

Table 3 Duration of chemosis in days in groups $G$ and $G W$

\begin{tabular}{llrrlll}
\hline \multirow{2}{*}{ Group } & \multicolumn{2}{l}{ Number of patients $(\%)$} \\
\cline { 2 - 7 } & 3 Days & 4 Days & 5 days & 6 Days & 7 Days & Total \\
\hline G & $4(17)$ & $6(26)$ & $9(39)$ & $1(4)$ & $3(13)$ & 23 \\
GW & $5(23)$ & $5(23)$ & $8(36)$ & $2(9)$ & $2(9)$ & 22 \\
Total & $9(20)$ & $11(24)$ & $17(38)$ & $3(7)$ & $5(11)$ & 45 \\
\hline
\end{tabular}

gentamicin injection in cataract surgery is widespread. Gentamicin has a wide spectrum of action against common ocular pathogens, and subconjunctival injections have been shown to provide effective levels of gentamicin in the aqueous in patients undergoing cataract surgery. ${ }^{1-4}$

Retinal toxicity has been reported with the use of intravitreal ${ }^{5}$ and accidental intraocular ${ }^{6}$ injec- $^{-}$ tion of gentamicin. Goulstine and Marmion in $1971^{7}$ reported on the tolerance of a single subconjunctival injection of $20 \mathrm{mg}$ gentamicin given to 10 patients at the conclusion of routine cataract surgery. They found that six of the patients had minimal conjunctival injection and chemosis 24 hours after the injection, which disappeared after a further 24 hours. They concluded that subconjunctival gentamicin is well tolerated locally and produces very little chemosis. In the present study 45 of 50 patients in the two groups (group G and GW) had chemosis. Of these 45 patients only $13(29 \%)$ had minimal or grade 1 chemosis while $32(71 \%)$ had more severe grade 2 or grade 3 chemosis. The chemosis lasted from three to seven days, average 4.64 days. It thus was not an insignificant problem, and in a few cases inpatient stay was longer than usual because of it.

The gentamicin preparation used in this study, gentamicin injectable $80 \mathrm{mg}$ in $2 \mathrm{ml}$ (Cidomycin), is widely used for the purpose of subconjunctival injections in this country. The manufacturers do not recommend the use of this preparation for subconjunctival use. Their recommended preparation for subconjunctival use in the prophylaxis prior to intraocular surgery is gentamicin sterile powder (Cidomycin), available in packs of $1 \mathrm{~g} .{ }^{8}$ The author believes this is not used as widely as the gentamicin injectable preparation. The difference between the two preparations is that gentamicin injectable has sodium metabisulphate, methyl hydroxybenzoate, propyl hydroxybenzoate, and disodium edetate as additives, while the recommended preparation gentamicin sterile powder has no additives.

Jenkins $e t a l^{9}$ in 1990 studied the comparative conjunctival toxicity of subconjunctival gentamicin and cefuroxime in a single-blind trial. They reported greater conjunctival toxicity in the form of hyperaemia, conjunctival oedema and capillary closure with subconjunctival gentamicin. The greater toxicity of gentamicin reported in their study may reflect the fact that the gentamicin preparation used, gentamicin injectable (Cidomycin), had the additives mentioned above, while the cefuroxime (Glaxo) preparation used in their study had no additives at all.

The possibility of the additives in the gentamicin preparation having a causative role in the conjunctival toxicity observed both in the present study and in the study by Jenkins $e t a l^{9}$ cannot be ruled out. A follow-up study to assess the role of additives in gentamicin toxicity is being conducted in this department.

The cause of postoperative conjunctival chemosis in our study was the subconjunctival injection of gentamicin given at the end of the cataract operation. The chemosis was significant 
in severity and duration and in some patients led to prolonged inpatient stay.

1 Furgiuele FP. Penetration of gentamicin into the aqueous humour of human eyes. Am $\mathcal{F}$ Ophthalmol 1970; 69: 4813 .

2 Mathalone MBR, Harden A. Penetration and systemic absorp tion of gentamicin after subconjunctival injection. $\mathrm{Br}$ Ophthalmol 1972: 56:609-12.

3 Jain MR, Bansal VD. Gentamicin penetration in human aqueous after subconjunctival injection. Indian $\mathcal{F}$ Ophthalmol 1981; 29: 307-11

4 Jain MR, Goyal M, Jain V. Ocular penetration of subconjunc- tivally injected gentamicin, sisomicin and cephaloridine. $\mathscr{f} p n$ f Ophthalmol 1988; 32: 392-400.

5 Conway BP, Campochiaro MD. Macular infarction after endophthalmitis treated with vitrectomy and intravitreal gentamicin. Arch Ophthalmol 1986; 104: 367-71.

6 McDonald HR, Schatz H, Allen AW, et al. Retinal toxicity secondary to intraocular gentamicin injection. Ophthalmology 1986; 93: 871-7.

7 Goulstine DB, Marmion VJ. Subconjunctival gentamicin. $\mathrm{Br} \mathcal{F}$ Ophthalmol 1971; 55: 478-80.

8 Roussel Laboratories Limited. Cidomycin Injectable data sheet. In: ABPI data sheet compendium. London: Datapharm Publications, 1990: 1470-1.

9 Jenkins CDG, McDonnell PJ, Spalton DJ. Randomised single blind trial to compare the toxicity of suconjunctival gentamicin and cefuroxime in cataract surgery. $\mathrm{Br} \mathcal{F}$ Ophthalmol 1990 74: $734-8$.

\section{FIFTY YEARS AGO}

\section{Ophthalmological 'moots' in the curriculum}

It has often struck us that more use might be made in the teaching of ophthalmology of gatherings at stated intervals, say once a week, at which a subject is propounded and discussed by the students. Such meetings used to be held, we believe, in the Mayo Clinic in general surgery, and each student in turn would propose some question for discussion. Something similar to the 'Moots,' those post-prandial meetings for the cracking of legal nuts, which were revived some years ago at the Inns of Court, might be attempted at the eye hospitals in Great Britain. Such a plan would give the student confidence and would help to balance the knowledge acquired in the week in question. Ophthalmology offers plenty of subjects for serious discussion and if a wag once in a way were to ask the question whether strabismus came to England with William the Conqueror or was indigenous among the aborigines; such a light-hearted theme, incapable of proof, might help to vary the monotony. The arrangements might be left as a rule in the hands of the chief clinical assistants, and a weekly rota of attendances for the seniors, who would occupy the position of judge or arbiter, would let each man know beforehand the date of his attendance and would not add very greatly to his labours.

At the present time when the question of post-war reconstruction is being much discussed, we commend this idea to those in authority for what it is worth. On a small scale the scheme would have some affinity to the ophthalmic club which has been for many years a feature among the seniors in the profession.

Annotation in BrF Ophthalmol 1941; 25: 535-6. 